\title{
Ocorrência de Telenomus alecto Crawford, 1914 (Hymenoptera: Scelionidae) em ovos de Diatraea spp. (Lepidoptera: Crambidae) em cana-de-açúcar no estado de Alagoas, Brasil
}

\author{
Occurrence of Telenomus alecto Crawford, 1914 (Hymenoptera: Scelionidae) \\ in eggs of Diatraea spp. (Lepidoptera: Crambidae) in sugar cane in the state \\ of Alagoas, Brazil \\ Nivia da Silva Dias ${ }^{1}$, Sônia Maria Forti Broglio-Micheletti ${ }^{1}$, Natália Rafaella de Moraes Farias ${ }^{1}$, \\ Simone Silva da Costa ${ }^{1}$, Jakeline Maria dos Santos ${ }^{1}$, Diego Olympio Peixoto Lopes ${ }^{1}$, \\ Valmir Antonio Costa ${ }^{2}$
}

\begin{abstract}
RESUMO
O objetivo deste trabalho foi verificar a ocorrência natural de T. alecto, parasitóide de ovos de Diatraea spp. em cana-de-açúcar no Estado de Alagoas. As posturas de D. flavipennella e D. saccharalis apresentaram 47,9\% e 30,0\% dos ovos com características de parasitismo e viabilidade de $92,4 \%$ e $89,9 \%$, respectivamente. A presença deste inimigo natural sugere um potencial de regulação natural de Diatraea spp.
\end{abstract}

Palavras-chave: Controle biológico, parasitoide de ovos, broca da cana-de-açúcar.

\section{ABSTRACT}

The objective of this study was to investigate the natural occurrence of T. alecto, an egg parasitoid of Diatraea spp. in sugar cane in the State of Alagoas. The eggs of $\mathrm{D}$. flavipennella and D. saccharalis presented $47.9 \%$ and $30.0 \%$ with characteristics of parasitism, and viabilities of $92.4 \%$ and $89.9 \%$, respectively. The presence of this natural enemy suggests a potential for natural regulation of Diatraea spp.

Key words: Biological control, egg parasitoid, sugar cane borer.

\section{Introdução}

O Brasil é o maior produtor e exportador de cana-de-açúcar no mercado internacional. A previsão de produção, para a atual safra, indica que o total dessa matéria-prima que será processada pelo setor sucroalcooleiro, deverá atingir um montante de 651,51 milhões de toneladas (Conab, 2009).

O maior produtor de cana-de-açúcar do Nordeste, em termos de área $(450,75 \mathrm{mil}$ ha de área cultivada) e produção é o estado de Alagoas. De acordo com o levantamento de produção para a safra 2009/2010, realizado pelo departamento técnico do Sindaçúcar-AL, houve uma produção de
24.270 milhões de toneladas de cana em Alagoas (Sindaçúcar-AL, 2011).

A cultura da cana-de-açúcar expande suas fronteiras a cada ano por todo o território nacional e com isso aumenta, consideravelmente, o risco de ataques de pragas. Algumas dessas se destacam pela frequência com que ocorrem e pelos prejuízos que causam, como é o caso das brocas do gênero Diatraea (Botelho et al., 1999). Diatraea saccharalis Fabricius, 1974 e D. flavipennella (Box, 1931) (Lepidoptera: Crambidae) são consideradas como as principais pragas da cana-de-açúcar, estando distribuídas em todas as regiões canavieiras do país. A primeira espécie encontra-se distribuída em todo

\footnotetext{
Embrapa Agroindústria Tropical, CEP: 60511-110 Fortaleza-CE, Brasil. nivia@cnpat.embrapa.br

Universidade Federal de Alagoas (CECA/UFAL), BR104, Km 85, CEP: 57100-00 Rio Largo-AL, Brasil.

E-mail: soniamfbroglio@gmail.com, simone_costa@hotmail.com, jackbilu@hotmail.com

Universidade Estadual Paulista “Júlio de Mesquita Filho”, CEP: 14.884-900 Jaboticabal-SP, Brasil.E-mail: diego_olympio@ hotmail.com

5 Instituto Biológico, CEP: 13012-970, Campinas-SP. E-mail: valmir@biologico.sp.gov.br
} 
o país, enquanto a segunda está restrita ao estado de Alagoas e a outros da região Nordeste (Freitas et al., 2006).

O principal método de controle para Diatraea spp. tem sido o biológico, empregando-se o endoparasitoide larval Cotesiaflavipes Cameron, 1891 (Hymenoptera: Braconidae), importado da Ásia na década de 1970. Embora este parasitoide seja um eficiente agente de controle de Diatraea spp., apresentando um custo de produção relativamente baixo, a exploração do potencial de parasitoides de ovos seria a forma ideal de controle biológico, uma vez que a fase de ovo é o fator chave para o crescimento populacional das brocas (Botelho et al., 1999).

Desta forma, este trabalho tem como objetivo registrar a ocorrência natural de parasitoides de ovos do gênero Telenomus (Hymenoptera: Scelionidae) em cana-de-açúcar sobre ovos de D. flavipennella e D. saccharalis, no estado de Alagoas.

O experimento foi conduzido em cultivo de cana-de-açúcar na área experimental do Centro de Ciências Agrárias (CECA) da Universidade Federal de Alagoas, município de Rio Largo, estado de Alagoas, Brasil (12 $40^{\prime} \mathrm{S}, 39^{\circ} 06^{\prime} \mathrm{W}, 127 \mathrm{~m}$ de altitude).

Para o levantamento da ocorrência natural dos parasitoides foi realizada uma infestação artificial com casais de adultos de $D$. saccharalis e $D$. flavipennella, provenientes da criação estoque do laboratório de Entomologia nos meses de setembro a novembro de 2008. Os casais foram confinados em gaiolas de nylon $(45 \mathrm{~cm} \times 95 \mathrm{~cm})$, dispostas nas folhas (estrato superior) para o acasalamento e oviposição.

A colocação das gaiolas foi realizada pela manhã, no intervalo entre 7:00 e 9:00h. No dia posterior, no mesmo horário, as gaiolas foram retiradas, os adultos eliminados e as posturas expostas ao parasitismo. Vinte e quatro horas após a retirada das gaiolas, as folhas com posturas foram levadas ao laboratório, as quais permaneceram acondicionadas em sala climatizada $\left(25 \pm 1{ }^{\circ} \mathrm{C}\right.$, UR $70 \pm 10 \%$, fotofase de $14 \mathrm{~h}$ ). As posturas que apresentavam o escurecimento característico foram monitoradas para a definição do parasitismo (número de posturas parasitadas/número total de posturas coletadas). Os exemplares adultos foram identificados e depositados na Coleção de Insetos Entomófagos "Oscar Monte" (IB-CBE), sediada no Centro Experimental do Instituto Biológico, em Campinas, SP. A identificação foi feita com base em Crawford (1914) e em Bin \& Johnson (1982).

Utilizaram-se 30 gaiolas para cada espécie de Diatraea spp. Cada gaiola foi constituída por 4 casais.
Com o intuito de verificar se os ovos de D. flavipennella e D. saccharalis, provenientes do campo, eram hospedeiros em potencial no desenvolvimento de T. alecto, avaliou-se a viabilidade (\% de posturas com orifícios de emergência), o número de indivíduos/ovo e a razão sexual dos descendentes.

De todas as coletas encaminhadas ao laboratório, foi registrado o total de 271 posturas de D. flavipennella e 233 de D. saccharalis. Dessas, 150 apresentaram ovos com características de parasitismo para D. flavipennella e 70 para $D$. saccharalis, o que correspondeu a $47,9 \%$ e $30,0 \%$, respectivamente. Os espécimes foram identificados como Telenomus alecto Crawford, 1914 (Hymenoptera: Scelionidae).

A viabilidade do material coletado em campo foi de $92,4 \%$ para D. flavipennella e $89,9 \%$ para D. saccharalis. Foi registrado apenas um indivíduo do parasitoide/ovo dos hospedeiros, e a razão sexual foi de $0,9 \mathrm{em}$ ambos os hospedeiros.

Espécies do gênero Telenomus apresentam variações quanto a preferência ao hospedeiro, mas parasitam principalmente ovos de lepidópteros. T. alecto é uma espécie encontrada em canaviais de diversos países da América do Sul e Central (Cueva \& Manuel, 1979; Terán 1980; Bin \& Johnson 1982) parasitando ovos de D. saccharalis e Diatraea rufescens Box, 1931. No Brasil essa espécie não é facilmente encontrada. Souza (1961) e Lima Filho et al. (1979) haviam encontrado T. alecto no Rio de Janeiro, mas a porcentagem de parasitismo por essa espécie era bem menor naquela região.

A constatação da ocorrência natural de T. alecto, e de seu potencial de parasitismo e viabilidade em D. flavipennella e D. saccharalis, mostra quão promissora é essa espécie no controle biológico de Diatraea spp. em cana-de-açúcar no estado de Alagoas.

Houve parasitismo de T. alecto sobre ovos das espécies de Diatraea, na área cultivada com canade-açúcar, sendo os ovos de $D$. flavipennella mais parasitados do que ovos de D.saccharalis.

\section{Agradecimentos}

Ao Conselho Nacional de Desenvolvimento Científico e Tecnológico $(\mathrm{CNPq})$ e à Fundação de Amparo à Pesquisa do Estado de Alagoas (FAPEAL) pelo apoio financeiro. A FITOSSAN (Assistência Fitossanitária e Controle Biológico LTDA) pelo fornecimento de material biológico. 


\section{Referências Citadas}

Bin, F \& N.F. Johnson.

1982 Potential of Telenominae in biocontrol with egg parasitoids (Hym., Scelionidae). Colloques del'INRA 9: 275-287.

Botelho, P.S.M., J.R.P. Parra, J.F. Chagas Neto \& C.P.B. Oliveira. 1999 Associação do parasitóide de ovos Trichogramma galloi Zucchi (Hymenoptera: Trichogrammatidae) e do parasitóide larval Cotesia flavipes (Cam.) (Hymenoptera: Braconidae) no controle de Diatraea saccharalis (Fabr.) (Lepidoptera: Crambidae) em cana-de-açúcar. Anais da Sociedade Entomológica do Brasil 28: 491-496.

CONAB (Companhia Nacional de Abastecimento)

Central de Informações Agropecuárias. Disponível em www. conab.gov.br. Acesso em junho de 2009.

Crawford, J.C.

1914 New parasitic Hymenoptera from British Guiana. Proceedings of the Entomological Society of Washington 16: 85-88.

Cueva, C \& A. M.

1979 Estudio preliminar de las poblaciones de huevos de Diatraea saccharalis (F.) y sus parásitos naturales en la caña de azúcar. Revista Peruana de Entomología 22: 25-28.

Freitas, M.R., A.P. Fonseca, E.L. Silva, A.L. Mendonça, C.E. Silva, A.L. Mendonça, R. Nascimento \& A.E. Santana.

2006 The predominance of Diatraea flavipennella (Lepidoptera: Crambidae) in sugar cane fields in the states of Alagoas, Brazil. Florida Entomologist 89: 539-540.

Lima Filho, M., G.M. Riscado, J.T. Barbosa.

1979 Comportamento da broca da cana-de-açúcar, Diatraea spp. no estado do Rio de Janeiro, no triênio 1975-77. Anais da Sociedade Entomologica do Brasil 8: 257-280.

Souza, H.D.

1961 Dois parasitos dos ovos da broca da cana, Diatraea saccharalis (Fabr., 1794). Divulgação Agronômica Shell, Rio de Janeiro 3: 1-5.

Terán, B.J.

1980 Lista preliminar de Hymenoptera parásitos de otros insectos en Venezuela. Revista de la Faculdad Agronomía 11: 283-389. 